\title{
Sotos Syndrome
}

National Institute of Neurological Disorders and Stroke (NINDS)

\section{Source}

National Institute of Neurological Disorders and Stroke (NINDS). Sotos Syndrome

Information Page.

Sotos syndrome (cerebral gig antism) is a rare genetic disorder caused by mutation in the NSD1 gene on chromosome 5. It is characterized by excessive physical growth during the first few years of life. Children with Sotos syndrome tend to be large at birth and are often taller, heavier, and have larger heads (macrocrania) than is normal for their age. Symptoms of the disorder, which vary among individuals, include a disproportionately large and long head with a slightly protrusive forehead and pointed chin, large hands and feet, hypertelorism (an abnormally increased distance between the eyes), and downslanting eyes. The disorder is often accompanied by mild cog nitive impairment; delayed motor, cognitive, and social development; hypotonia (low muscle tone), and speech impairments. Clumsiness, an awkward gait, and unusual aggressiveness or irritability may also occur. Although most cases of Sotos syndrome occur sporadically (meaning they are not known to be inherited), familial cases have also been reported. 